\title{
Where are the Fermi Lines Coming From?
}

\author{
Kanishka Rao ${ }^{1}$ and Daniel Whiteson ${ }^{1}$ \\ ${ }^{1}$ Dept. of Physics 83 Astronomy, UC Irvine, Irvine, CA
}

\begin{abstract}
We estimate the spatial locations of sources of the the observed features in the Fermi-LAT photon spectrum at $E_{\gamma}=110$ and $E_{\gamma}=130 \mathrm{GeV}$. We determine whether they are consistent with emission from a single source, as would be expected in their interpretation as $\gamma \gamma$ and $\gamma Z$ lines from dark matter annhiliation, as well as whether they are consistent with a dark matter halo positioned at the center of the galaxy. We take advantage of the per-photon measured incident angle in reconstructing the line features. In addition, we use a data-driven background model rather than making the assumption of a feature-less background. We localize the sources of the features at 110 and $130 \mathrm{GeV}$. Assuming an Einasto (NFW) density model we find the $130 \mathrm{GeV}$ line to be offset from the galactic center by $285(280) \mathrm{pc}$, the $110 \mathrm{GeV}$ line by 60 (30) pc with a large relative separation of 220 (240) pc. However, we find this displacement of each source from the galactic center, as well as their relative displacement to be statistically consistent with a single Einasto or NFW dark matter halo at the center of the galaxy.
\end{abstract}

PACS numbers:

\section{INTRODUCTION}

The search for the particle nature of dark matter is one of the oustanding open questions of modern physics. A broad program of research with a variety of promising and complementary approaches has become a major piece of experimental particle physics research. This includes searches for direct production at colliders, searches for dark matter interactions with standard model particles in large quiet underground detectors, and searches for signals of dark matter annhiliation into standard model particles in regions of the galaxy with large dark matter density.

A clear signal of dark matter annihilation may be carried by gamma rays traveling to Earth from regions in the galaxy with high dark-matter density. As the photons do not typically scatter after their production, the photon energy and direction are powerful handles for understanding the mechanism of dark matter annihiliation into standard model particles.

One mechanism is annihilation resulting in quarks, which would hadronize and yield $\pi^{0}$ particles which in turn produce photons. The broad spectrum of such a process would give fairly low energy photons $\left(E_{\gamma} \lesssim 50 \mathrm{GeV}\right)$ and may be difficult to distinguish from other sources.

A clearer signature may appear from annihilation directly into two-body final states including a photon, though the rate would be smaller than continuum emission due to loop supression. Rather than yielding a broad energy spectrum, this process would produce a photon with a well-defined energy (a "line"). This makes a search for lines in the photon spectrum an important component of the dark matter program using Fermi-LAT data [1] 3 .

Recently, observation of a feature with high local statistical significance at $E_{\gamma}=130 \mathrm{GeV}$ was reported [4, 5], with a source location 1-2 degrees away from the galactic center [6]. Follow-up analyses suggested the possibility of two features, consistent with the spacing of lines expected from $\gamma \gamma$ and $\gamma Z$ processes [7, 8,

In this paper, we study in detail the question of the location of the source of the photons in the features. We confirm the location of the photons in the feature at $E_{\gamma}=130 \mathrm{GeV}$, and for the first time, locate the source of the photons in the feature at $E_{\gamma}=110 \mathrm{GeV}$. We study whether the two are - individually or collectively - consistent with emission from a single source, as would be expected if they represent the $\gamma \gamma$ and $\gamma Z$ processes, as well as whether that source is consistent with a dark matter halo at the galactic center. In addition, we introduce a new approach to analyzing the data which uses the measured angles of the individual photons, rather than the median photon angles.

\section{ENERGY SPECTRUM ANALYSIS}

A source of photons with a very narrow range of energies would appear in the spectrum of observed FermiLAT photons as a peak, due to finite energy resolution. Previous analyses [4, 7, 8, have used the Fermi-LAT energy dispersion formula 9 to build a probability density function $(p d f)$ for the reconstructed energies of photons from a line, and fit it to the observed spectrum.

Given the small size of the dataset, we seek to use the maximum available information about each photon to extract insight into the observed features. The energy resolution, for example, has a significant dependence on two other photon characteristics: $\theta$, the incident angle relative to a line normal to the LAT face, and the photon 'type' which indicates whether the conversion happened in the front or back layers of the tracker [10]. Figure 1 
shows the reconstructed energy pdf for various choices of $\theta$ and type. This per-photon information is very pertinant to the analysis of this spectrum. If the photons in the peak were all well-measured, it would enhance the significance of the peak; conversely, if they were all poorlymeasured, it would degrade the significance.

Our approach is to reconstruct the distribution of $E_{\gamma}^{\text {true }}$, the true energy of photons striking the LAT, rather than the distribution of $E_{\gamma}^{\text {reco }}$, the reconstructed energy of each photon. The former distribution can account for the per-photon resolution, while the latter treats each photon equally. The reconstructed energy is related to the true energy by the pdf $f\left(E^{\text {reco }} \mid E^{\text {true }}, \theta\right.$, type $)$. We use Bayes theorem to invert this relation and calculate $f\left(E^{\text {true }} \mid E^{\text {reco }}, \theta\right.$, type $)$ where $P\left(E^{\text {reco }}\right)$ is taken from the full-sky photon energy spectrum. Other choices of priors have negliglible effect, as the energy dispersion pdf is sharply peaked, see Fig 1 .

The distribution of $E_{\gamma}^{\text {true }}$ is then built as a kernel density estimate 11 with $f\left(E^{\text {true }} \mid E^{\text {reco }}, \theta\right.$, type $)$ as the choice of kernel. Other approaches [5, 6] have used adaptive Gaussian kernels, but our physically motivated choice of kernel reflects the asymmetric shape of the pdf and uses - for the first time - the per-photon measured incident angle rather than the median angle of the dataset.

We use the publicly available Fermi-LAT data collected over 3.7 years. We use ultraclean photons with zenith angle $<100$ to veto photons from Earth as well as standard quality requirements [12. We select a three-degree region around the galactic center.

The observed photon counts in the three-degree region are shown in Fig. 2. The distribution of $E_{\gamma}^{\text {true }}$ is shown in Fig. 3. with several choices of angle-dependence used to demonstrate the impact of this information. Two choices demonstrates the range of possibilities, if all photons had the maximum or minimum possible energy resolutions. This demonstrates the potential importance of this information. The spectrum which uses the measured perphoton angles has peaks at 110 and $130 \mathrm{GeV}$ which are slightly degraded relative to a spectrum which uses the median photon angle. As the distribution of photons in the peak region and the background region have similar distributions of $\cos (\theta)$ and conversion type [13, there is not currently a large impact from using the per-photon information, but it remains a useful method to visualize the spectrum.

\section{LOCATING THE FEATURES}

\section{Technique}

We search for the most likely source position using a maximal likelihood fit of the observed photons to the expected spatial and energy distributions from dark matter halos. The unbinned likelihood is
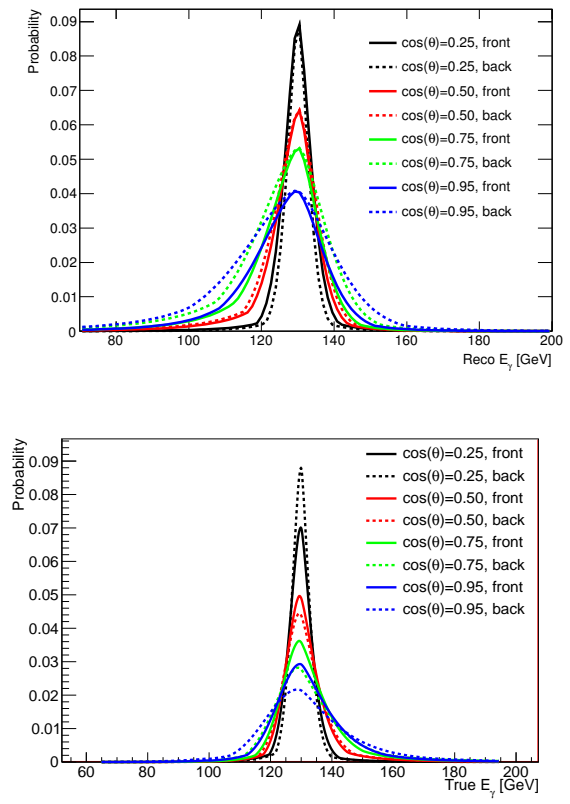

FIG. 1: Probability density functions which describe the energy dispersion of the Fermi-LAT. Top, the probability density for reconstructed photon energies for a photon with $E_{\gamma}^{\text {true }}=130 \mathrm{GeV}$. Bottom, the probability density for true photon energies for a photon with $E_{\gamma}^{\text {reco }}=130 \mathrm{GeV}$.

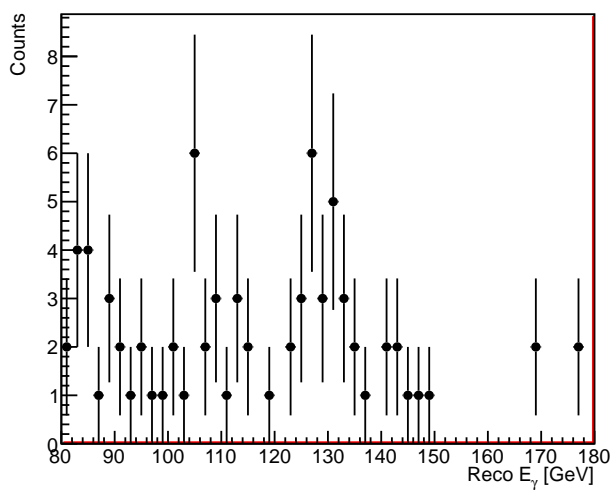

FIG. 2: Reconstructed photon energies $E_{\gamma}^{\text {reco }}$ from FermiLAT in a three-degree region surrounding the galactic center.

$$
L(l, b)=\prod_{i}^{N_{i}} \frac{n_{\mathrm{bg}} f_{\mathrm{bg}}\left(E_{i}, \theta_{i}\right)+n_{\mathrm{sig}} f_{\mathrm{sig}}\left(E_{i}, l_{i}, b_{i} \mid \theta_{i}, l, b\right)}{n_{\mathrm{bg}}+n_{\mathrm{sig}}}
$$

where $(l, b)$ are the galactic coordinates of the center of the dark matter halo, $n_{\mathrm{bg}}=$ and $n_{\text {sig }}$ are the background and signal normalizations, the index $i$ runs over observed photon energy and spatial parameters $\left(E_{i}, \theta_{i}, l_{i}, b_{i}\right)$ within the energy window surrounding the 


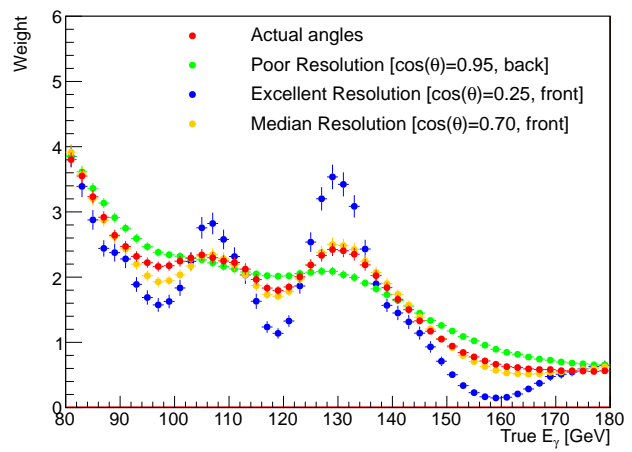

FIG. 3: Relative likelihood of true photon energies, $E_{\gamma}^{\text {true }}$, built from the reconstructed energies of Fig 2 and the pdf $f\left(E^{\text {true }} \mid E^{\text {reco }}, \theta\right.$, type $)$, see text for details. The impact of different assumptions about the photon incident angle $\theta$ is shown.

feature(s) of interest, and $f_{\text {sig }}\left(E_{i}, l_{i}, b_{i} \mid \theta_{i}, l, b\right)$ is the dark matter halo pdf as a function of photon energy $E_{i}$, incidence angle $\theta_{i}$ at spatial position $\left(l_{i}, b_{i}\right)$ given a dark matter halo centered at $(l, b)$. The pdf $f_{\text {sig }}$ accounts for per-photon spatial [14] and energy [9] resolution in the same spirit as the Bayesian unfolding described above; here we do not need to explicitly unfold, as we have included the per-photon information in the unbinned likelihood.

The background pdf $f_{\mathrm{bg}}$ is built from a data-driven model, with the energy dependence coming from photons outside the three-degree region surrounding the galactic center, see Figure 4. We find this to be reasonably consistent with a power law model used in previous analyses [4 8]. We use energy windows of $E_{\gamma}^{\text {true }}=$ $[105,115],[125,135]$ for the location of the feature at 110 $\mathrm{GeV}$ and $130 \mathrm{GeV}$, respectively, or both windows for the combined features. In the case of the two-feature analysis $\left(E_{\gamma}=110,130 \mathrm{GeV}\right)$, there are two $f_{\text {sig }}$ terms in the likelihood, one for each peak. We use normalizations of $n_{\text {sig }}=6$ and $n_{\text {sig }}=14$ for the $E_{\gamma}=110$ and $130 \mathrm{GeV}$ features, respectively.

The dark matter halo pdfs $f_{\text {sig }}$ are derived from either NFW [15] or Einasto [16] halo profiles; the pdf in $(l, b)$ is calculated via the line-integral of the square of the dark matter density [17]. We use $\alpha_{E}=0.17$ for the Einasto model and $r_{s}=20 \mathrm{kpc}$ for both models.

\section{Results}

The position of the most likely values of the halo cen-

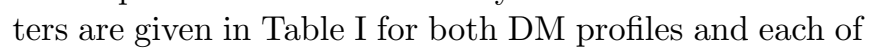
the features as well as the combined spectrum. In both cases, the feature at $130 \mathrm{GeV}$ appears to be displaced from the galactic center, as previously reported, but the

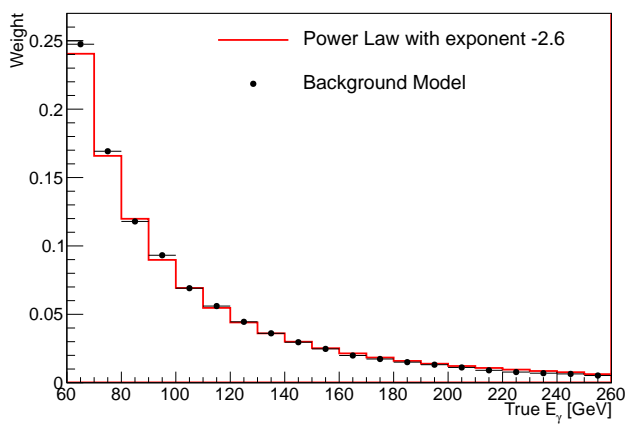

FIG. 4: The data-driven background model (points) constructed from photons outside the three-degree region surrounding the galactic center, compared to a power law with exponent -2.6 .

$110 \mathrm{GeV}$ feature appears to be centered at the galactic origin.
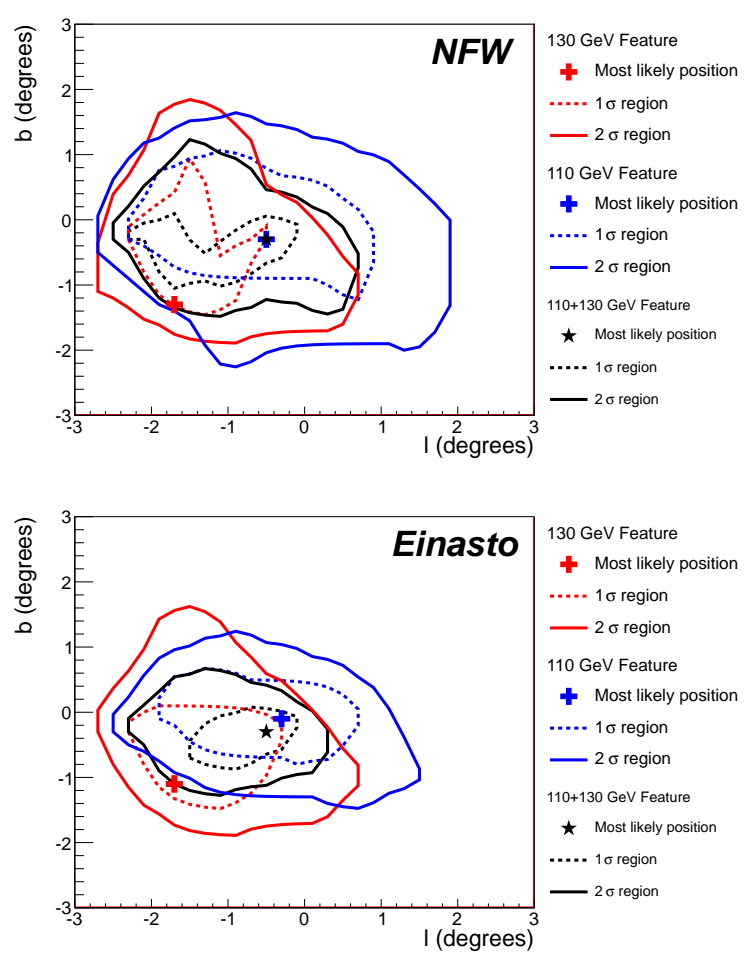

FIG. 5: Most likely positions in galactic coordinates $(l, b)$ of an NFW (top) or Einasto (botton) dark matter halo, maximized seperately for the features at $130 \mathrm{GeV}, 110 \mathrm{GeV}$, or the combined features. Also shown are $1 \sigma$ and $2 \sigma$ uncertainty regions, see text for details.

Figure 5 shows the most likely positions for each of the dark matter halo profiles and each of the energy spectrum features, as well as uncertainty regions. The uncertainty regions are calculated in a frequentist man- 
TABLE I: Most likely positions in galactic coordinates $(l, b)$ of an NFW or Einasto dark matter halo, maximized seperately for the features at $130 \mathrm{GeV}, 110 \mathrm{GeV}$, or the combined features.

\begin{tabular}{lccc}
\hline \hline & $130 \mathrm{GeV}$ & $110 \mathrm{GeV}$ & Combined \\
\hline NFW & $(-1.5,-1.1)$ & $(-0.2,0.1)$ & $(-0.4,-0.2)$ \\
Einasto & $(-1.5,-1.2)$ & $(-0.4,-0.2)$ & $(-0.2,0.1)$ \\
\hline \hline
\end{tabular}

ner, using simulated experiments to determine the $\Delta L$ threshould which would contain the true $(l, b)$ position in $68 \%$ (for $1 \sigma$ ) or $95 \%$ (for $2 \sigma$ ) of the cases.

If the two features were both due to dark matter annhililation processes, one would expect them to be colocated. Figure 6 shows the unfolded $E_{\gamma}^{\text {true }}$ spectrum in a 1-degree circle surrounding each of the features; in the spectrum which highlights the $130 \mathrm{GeV}$ feature, the 110 $\mathrm{GeV}$ feature is suppressed, and vice versa. Similarly, Figure 7 shows the unfolded $E_{\gamma}^{\text {true }}$ spectrum in the $1 \sigma$ region surrounding each feature.
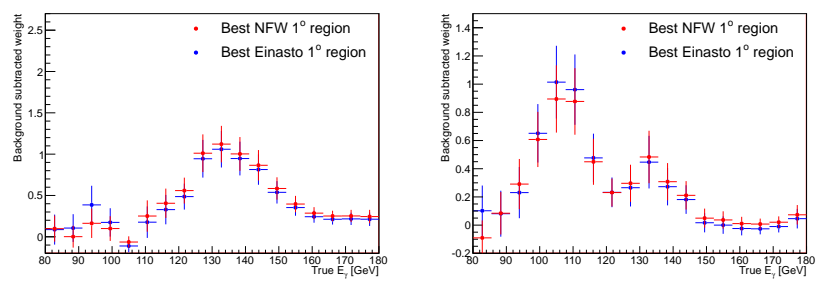

FIG. 6: Spectrum in a region within 1 degree of the maximum likelihood for 130 (left) and 110 (right) features assuming an Einasto or NFW source.
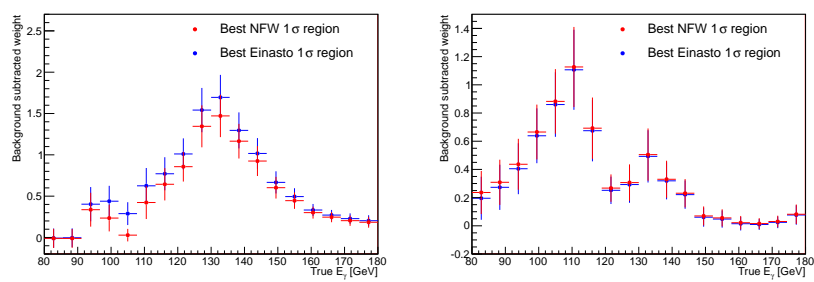

FIG. 7: Spectrum in a region within $1 \sigma$ of the maximum likelihood for 130 (left) and 110 (right) features assuming an Einasto or NFW source.

This suggests that the two features of the energy spectrum may have spatially distinct sources, and these sources may themselves be offset from the galactic center. To evaluate the significance of the sources' displacement from the galacter center and from each other a more rigorous analysis which accounts for uncertainties is performed below.

\section{Interpretation}

If the observed features are both due to dark matter annhilation, then their locations in the sky depend only on the dark matter density and not the nature of the final state, $\gamma \gamma$ or $\gamma Z$, and so should yield locations consistent with a single source. As shown above, we find that the sources of the $E_{\gamma}=110$ and $130 \mathrm{GeV}$ features are seperated appreciably from each other, and that the $E_{\gamma}=130$ feature is displaced from the galactic center.

Definitive conclusions, however, require analysis of the uncertainties involved, including the expected fluctuations of the measured source locations given the small number of photons in each feature. We seek to answer two questions:

- Are the two features consistent with emission from a single source?

- Are the features consistent with emission from a dark matter halo at the galactic center?

We answer these questions by comparing hypotheses using likelihood ratios. For example, we can probe whether the feature at $E_{\gamma}=130 \mathrm{GeV}$ is consistent with emission from the galactic center by evaluating

$$
q=-2 \log \frac{L(l=\hat{l}, b=\hat{b})}{L(l=0, b=0)}
$$

where $(\hat{l}, \hat{b})$ is the location which maximizes $L$ and $(0,0)$ serves as the null hypothesis. When $(\hat{l}, \hat{b})$ is close to $(0,0)$, the $\log$ of the likelihood ratio $q$ approaches zero; when it is far from the origin, it becomes negative. We compare the measured value of $q$ to the expected distribution in simulated experiments from an NFW or Einasto profile with a line at $E_{\gamma}=130 \mathrm{GeV}$ placed at $(0,0)$, see Figures 8(a) and 9(a). While the value of $q$ indicates that the maximal position is displaced from the origin, it is consistent with the expected distribution of $q$ values when the DM halo is at the origin; the $p$-values $=0.18$ and 0.14 for Einasto or NFW, respectively. Distributions of $q$ for DM halos offset from the origin are also shown.

We perform this analysis for the feature at $E_{\gamma}=110$ $\mathrm{GeV}$ as well, see Figures 8(b) and 9(b). As this feature appears to be located close to $(0,0)$, the $q$ values are close to zero and entirely consistent with a DM halo at the galactic center.

We repeat the analysis for the combined features at 110 and $130 \mathrm{GeV}$, where we constrain them to have the same location, see Figures 8(c) and 9(c). Again, the $q$ values are consistent with a DM halo at the galactic center.

To address the question of whether the two features are more likely to be from a single source (consistent with a DM interpretation) or from two spatially distinct sources, we construct a likelihood ratio 


$$
q=-2 \log \frac{L_{130}(\hat{l}, \hat{b}) \times L_{110}(\hat{l}, \hat{b})}{L_{130}\left(\hat{l}_{130}, \hat{b}_{130}\right) \times L_{110}\left(\hat{l}_{110}, \hat{b}_{110}\right)}
$$

where in one case we find the single location $(\hat{l}, \hat{b})$ which maximizes $L$ for both features, and in the other we allow the two features to find individual maxima: $\left(\hat{l}_{110}, \hat{b}_{110}\right)$ and $\left(\hat{l}_{130}, \hat{b}_{130}\right)$. This $q$ will be zero if the individually optimized (and possibly separated) locations are consistent with the most likely single joint location, and positive if individually optimized (and possibly separated) locations give a better description of the data. Again we compare to the expected distribution of the likelihood ratio in simulated experiments in which the two sources are co-located and find that the observed $q$ is consistent with a co-located source, see Figures 8(d) and 9(d).
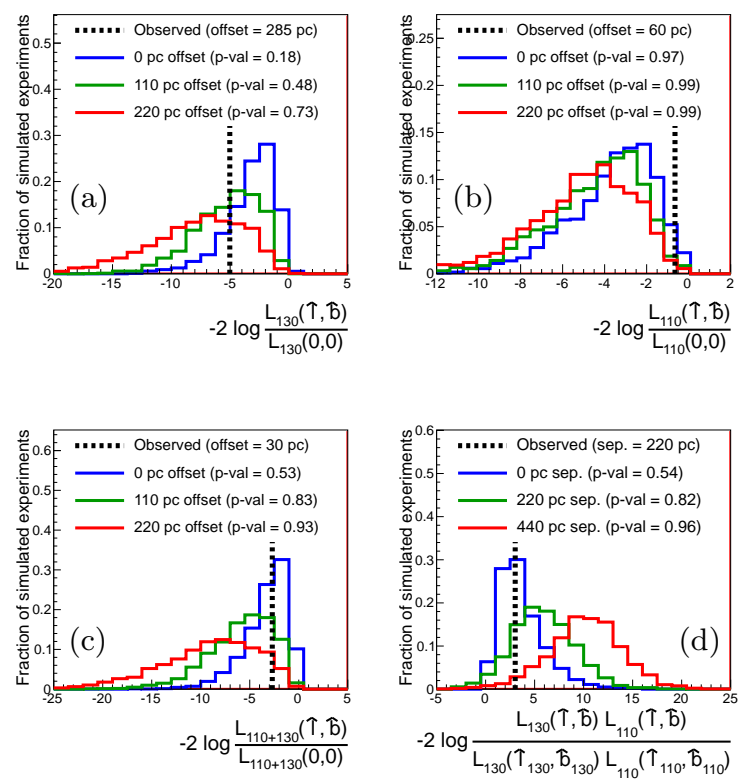

FIG. 8: The log of likelihood ratios comparing the likelihood of a Einasto DM halo placed at the most likely position $(\hat{l}, \hat{b})$ or at the galactic center $(l=0, b=0)$, in the case of the 130 $\mathrm{GeV}$ feature (a), the $110 \mathrm{GeV}$ feature (b), or the combined features (c). Also shown is the likelihood ratio between two co-located sources or sources which are seperated (d). In each case, we show the expected distribution in simulated experiments.

\section{CONCLUSION}

We haved demonstrated via Bayesian unfolding the potential importance of including the per-photon energy resolution, though for this dataset the results are little
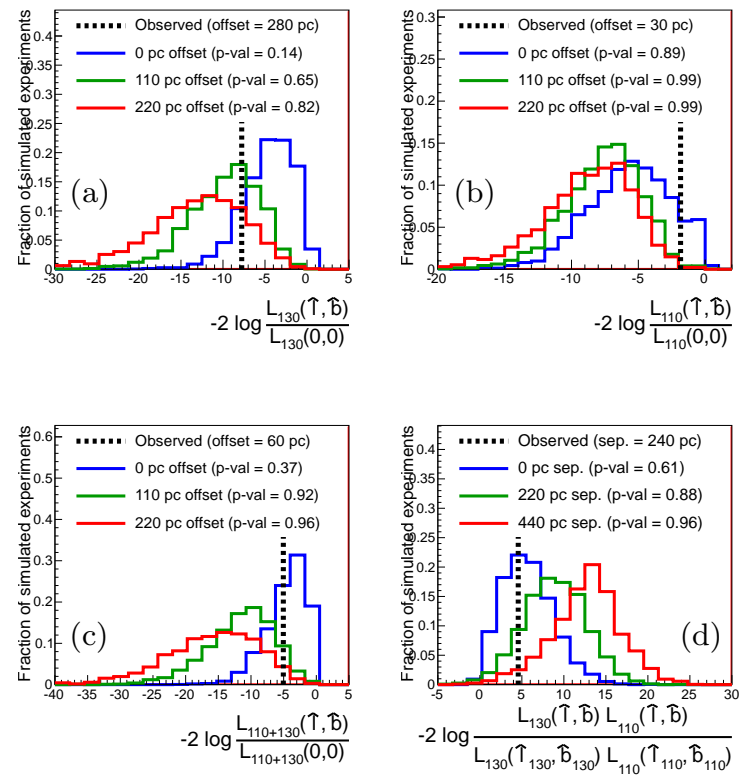

FIG. 9: The log of likelihood ratios comparing the likelihood of an NFW DM halo placed at the most likely position $(\hat{l}, \hat{b})$ or at the galactic center $(l=0, b=0)$, in the case of the $130 \mathrm{GeV}$ feature (a), the $110 \mathrm{GeV}$ feature (b), or the combined features (c). Also shown is the likelihood ratio between two co-located sources or sources which are seperated (d). In each case, we show the expected distribution in simulated experiments.

different from the standard approach of assuming the median angle and therefore resolution.

We have presented an analysis of the spatial locations of the sources of the observed features in the Fermi-LAT energy spectrum at $E_{\gamma}=110$ and $130 \mathrm{GeV}$. We find:

- the $130 \mathrm{GeV}$ photons come from a location displaced from the galactic center, but consistent with an Einasto or NFW dark matter halo at the center. This is consistent with previous work [17].

- the $110 \mathrm{GeV}$ photons come from a location close to the galactic center, and consistent with an Einasto or NFW dark matter halo at the center.

- the two features at 110 and $130 \mathrm{GeV}$ photons are consistent with a single DM halo at the galactic center, with either an Einasto or NFW density profile.

Some have suggested that the displacement of the source of the $E_{\gamma}=130 \mathrm{GeV}$ feature indicates that DM halos may be displaced from the galactic center 18, but we find that the current data are consistent with a DM halo from the center. 


\section{ACKNOWLEDGEMENTS}

The authors acknowledges contributions, explanations and useful discussions with Eric Albin which are clearly deserving of authorship, clever statistical insight from Kyle Cranmer, useful comments from Annika Peter, and technical support from Mariangela Lisanti and Tracy Slatyer. DW and KR are supported by grants from the Department of Energy Office of Science and by the Alfred P. Sloan Foundation. DW is grateful to the Aspen Center for Physics, where a portion of this work was performed and supported by NSF grant no. 1066293.

[1] M. Ackermann et al. [Fermi-LAT Collaboration], Phys. Rev. Lett. 107, 241302 (2011) arXiv:1108.3546 [astroph.HE]];

[2] A. A. Abdo et al. [ The Fermi-LAT Collaboration], Phys. Rev. Lett. 104, 091302 (2010) arXiv:1001.4836 [astroph.HE]].

[3] M. Ackermann et al. [Fermi-LAT Collaboration], arXiv:1205.2739 [astro-ph.HE].

[4] C. Weniger, arXiv:1204.2797 [hep-ph].

[5] E. Tempel, A. Hektor and M. Raidal, arXiv:1205.1045 [hep-ph].

[6] M. Su and D. P. Finkbeiner, (2012), arXiv:1206.1616
[7] A. Rajaraman, T. M. P. Tait and D. Whiteson, JCAP, accepted (2012), JCAP 1209, 003 (2012) arXiv:1205.4723 [hep-ph]].

[8] T. Cohen, M. Lisanti, T. R. Slatyer and J. G. Wacker, arXiv:1207.0800 [hep-ph].

[9] http://fermi.gsfc.nasa.gov/ssc/data/analysis/ documentation/Cicerone/Cicerone_LAT_IRFs/IRF_E_ dispersion.html

[10] http://fermi.gsfc.nasa.gov/ssc/data/analysis/ documentation/Cicerone/Cicerone_Data/LAT_Data_ Columns.html

[11] L. Holmström, H. Miettinen, and S. R. Sain, Comp. Phys. Comm. 88 (1995) 195-210.

[12] Pass7, ultraclean class, quality requirements: DATA_QUAL $=1$ \&\& LAT_CONFIG=1 \&\& ABS (ROCK_ANGLE) $\leq 52$

[13] D. Whiteson, arXiv:1208.3677 [astro-ph.HE].

[14] http://fermi.gsfc.nasa.gov/ssc/data/analysis/ documentation/Cicerone/Cicerone_LAT_IRFs/IRF PSF.html

[15] J. F. Navarro, C. S. Frenk and S. D. M. White, Astrophys. J. 462, 563 (1996) astro-ph/9508025.

[16] J. Einasto and U. Haud , Astron. Astrophys. 223, 89 (1989); A. W. Graham, D. Merritt, B. Moore, J. Diemand and B. Terzic, Astron. J. 132, 2685 (2006).

[17] R. -Z. Yang, Q. Yuan, L. Feng, Y. -Z. Fan and J. Chang, Phys. Lett. B 715, 285 (2012)

[18] M. Kuhlen, J. Guedes, A. Pillepich, P. Madau and L. Mayer, arXiv:1208.4844 [astro-ph.GA]. 